\title{
Dynamic behaviors of fractal-like domains in monolayers
}

\author{
Mu Wang, ${ }^{1}$ Cheng Sun, ${ }^{1}$ Ru-Wen Peng, ${ }^{1}$ Nai-Ben Ming, ${ }^{1}$ Jan van Esch, ${ }^{2 *}$ Helmut Ringsdorf, ${ }^{2}$ and Piet Bennema ${ }^{3}$ \\ ${ }^{1}$ National Laboratory of Solid State Microstructures, Nanjing University, Nanjing 210093 \\ and Center for Advanced Studies of Science and Technology of Microstructures, Nanjing 210093, China \\ ${ }^{2}$ Institute of Organic Chemistry, University of Mainz, D-55099 Mainz, Germany \\ ${ }^{3}$ Department of Solid State Chemistry, University of Nijmegen, 6525 ED Nijmegen, The Netherlands
}

(Received 3 August 1995)

\begin{abstract}
In this paper we report our recent investigations on the morphological evolution of fractal-like domains of the liquid-condensed (LC) phase in lipid monolayers. It is demonstrated that the dimension of the LC domains increases upon continuous illumination of microscope light. The experimental data indicate that the increasing rate of fractal dimension of the LC domains depends on the concentration of fluorescence probes. By analyzing the domain growth process we find that the self-similarity of the pattern disappears gradually during its growth. The possible mechanism behind the observed phenomena is discussed. [S1063-651X(96)00206-1]

PACS number(s): 81.10.Aj, 61.43.Hv, 64.60.-i, 68.10.-m
\end{abstract}

\section{INTRODUCTION}

Monolayers on the air-water interface provide an ideal two-dimensional (2D) system to study pattern formations [1-3]. By introducing fluorescence microscopy [1] and Brewster angle microscopy [4], the first-order transition from a liquid-expanded (LE) phase to a liquid-condensed (LC) phase can be directly observed. In many cases, domains of the LC phase possess fractal-like morphology $[1,3]$. The mechanism of the growth of fractal-like domains, as well as the nucleation and evolution process of LC domains, has been studied previously $[3,5-7]$. It has been reported recently that in monolayers the morphology of the LC domains may develop from compact faceted pattern to dendrite and fractals, or from fractal to nonfractal morphology [8]. Similar phenomenon occurs in a liquid crystal system where morphological transitions from a dense-branching pattern to dendrite to a dense-branching pattern have been observed as a function of temperature in the nematic phase of $8 \mathrm{CB}$ [9]. Ben-Jacob and Garik [10], Wang and Ming [11], and Hill [12] have proposed some selection hypotheses on how a specific pattern is selected. Cladis et al. have also studied the wavelength selection of a growing solid-liquid interface in solidification and tried to understand whether the wavelength selection is an intrinsic property or rather depends on boundary effects of the system [13]. However, for different experimental systems, the situation may vary significantly. At least in a monolayer system, the knowledge of pattern transitions is very limited and it seems that a universal law of pattern selection is still to be discovered.

In situ microscopy is a useful tool to investigate morphological evolution. Previously it was not easy to apply this technique to a Langmuir monolayer system. In conventional Langmuir monolayer experiments, the monolayer film on the air-water interface is compressed by a Teflon barrier to reach the LC phase. During the compression, convection always occurs in the water subphase. Therefore it was difficult to

\footnotetext{
*Present address: Department of Chemistry, Groningen University, 9747AG Groningen, The Netherlands.
}

trace a growing domain over a period longer than about tens of seconds [3]. By introducing an inhomogeneous electric field, it was possible to collect domains under a microscope and study their evolving process [14]. However, the applied electric field may influence the molecular aggregation dynamics [15] since the electric dipole-dipole interaction is believed to play an important role in the growth of LC domains. Recently, a different method has been developed which allows us to trace a specific domain continuously for a long period on the air-water interface $[8,16,17]$. A circular Teflon mask (diameter $20 \mathrm{~mm}$ ) is placed under the objective of the microscope in the water subphase. The wall of the mask is slightly higher than the water surface. The water surface within the Teflon mask is connected with the outside through an opening on the wall. Therefore, there should be no pressure difference over the monolayer film within and outside the mask when equilibrium is reached. By introducing the mask, surface flow in the plane of monolayer was suppressed greatly. Hence it is possible to study the growth behavior of a selected LC domain. In this paper, we demonstrate our studies on the phenomenon that the dimension of the fractal-like LC domains may increase gradually upon continuous illumination of the microscope light. We also find that the increasing rate of the fractal dimension of the LC domains varies as a function of the concentration of the fluorescence probe.

\section{EXPERIMENT}

The domain growth behavior is studied in the monolayer of imidazole surfactant R-(-)-N, N-dihexadecy-[2-(1imidazolyl)-propyl]amine, which was synthesized by one of the authors (J. van Esch). The synthesized imidazole surfactant was purified by column chromatography and then dissolved in chloroform (P.A. grade, Merck). $0.5 \% \mathrm{~mol}$ of DPPE-sulforhodamine (Molecular Probes, U.S.A.) was added as a fluorescent probe. The mixed solution was spread carefully onto the water surface in a Teflon-coated trough $\left(350 \mathrm{~cm}^{2}\right)$ in order to form a monolayer film. The whole trough was thermostated and placed on the stage of a fluorescence microscope. The water in the trough (PH 5.5) was purified by a Millipore system. The trough was equipped with a motorized Teflon barrier, which allows us to compress 

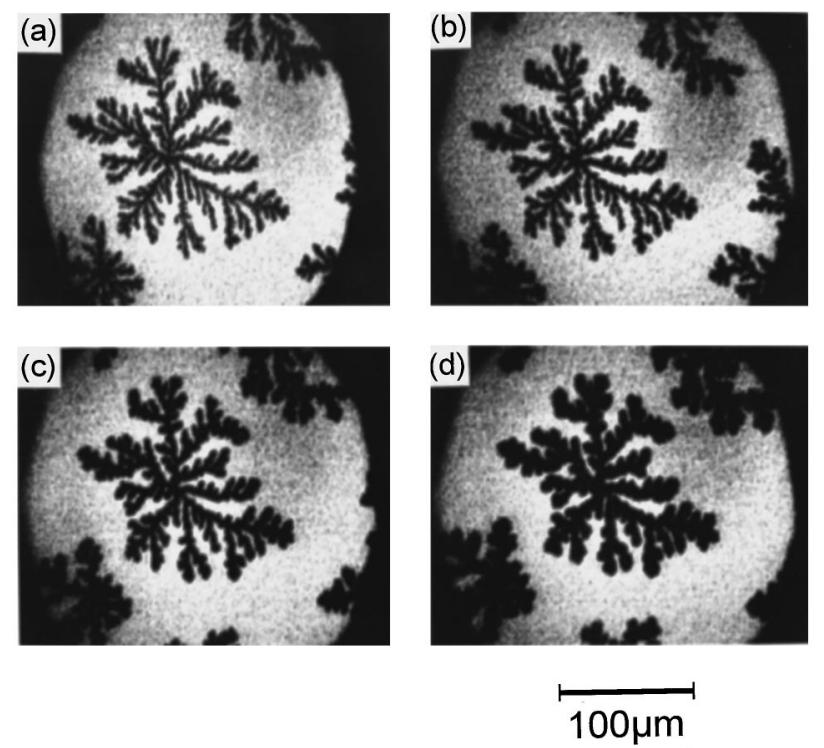

FIG. 1. The growing process of LC domains under continueous illumination of the microscope light. Meanwhile compression of the monolayer film has been stopped for a long period and the average area per molecule over the monolayer film is kept constant. One may notice that the morphology of the domain is thickened gradually from (a) to (d). The time interval between each frame is about 2 mintues. The area of the dark LC domain in the central part of each frames is (a) $6874 \mu \mathrm{m}^{2}$; (b) $7875 \mu \mathrm{m}^{2}$; (c) $8671 \mu \mathrm{m}^{2}$; (d) $9395 \mu \mathrm{m}^{2}$.

monolayers with an adjustable rate. The surface pressurearea $(\Pi-A)$ isotherm of the monolayers was measured simultaneously during the compression. The surface pressures were detected by Wilhelmy balances and were accurate to within $0.1 \mathrm{mN} \mathrm{m}^{-1}$. The temperature of the water subphase was kept at $20 \pm 0.1^{\circ} \mathrm{C}$. Optical filters were used to generate quasimonochromatic light $(\lambda=510 \sim 560 \mathrm{~nm})$ for the excitation of the fluorescent molecules. The trough, Wilhelmy balance, and temperature measuring system were all interfaced

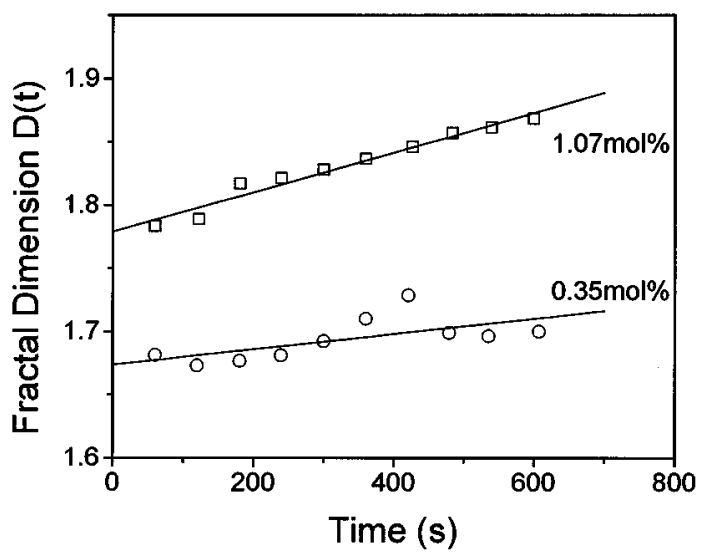

FIG. 2. The plot to show the evolution of the fractal dimension of the LC domain as a function of time. The dimension of the illuminated cluster increases gradually. One may find that the increasing rate of the fractal dimension becomes higher when the concentration of the fluorescence probe is higher. The fractal dimensions were determined by the method of the density-density correlation function. to a personal computer for data acquisition and system control. The domain growing process was recorded by a microscope-matched video system. The quantitative data of the domain growth, such as the increasing rate of the fractal dimension of the solid domains, etc., were obtained thereafter by image analysis.

When a homogeneous monolayer film was formed, we compressed the monolayer to the LE-LC coexistence region at a rate of several $\AA^{2}$ molecule ${ }^{-1} \mathrm{~min}^{-1}$. In our system, the LE-LC coexistence region was characterized by the formation of dark, fractal-like LC domains $[8,16,17]$. When the LC domains grew sufficiently large, the barrier was stopped. The domains ceased growing when an equilibrium surface pressure was reached. Thereafter, a LC domain was selected and kept at the center of the view field of the microscope for continuous observation. The optical field diaphragm (OFD) of the microscope was adjusted to get a suitable size of the shade of the OFD. Those domains in the shade of the OFD were protected from illumination and did not grow.

\section{RESULTS AND DISCUSSION}

Being continuously illuminated by the microscope light, the LC domains grow gradually, as shown in Fig. 1. This phenomenon is unusual because the mean time compression of the monolayer film has been completed for a long period and the average area per molecule (or average molecular density) was kept constant. From Fig. 1 one may find that the main branches of the fractal-like LC domain are thickened gradually and some minor branches shrink due to the effect of line tension. Image analyzing indicates that the area of the dark LC domain really increases from Fig. 1(a) to 1(d). This morphological transition may not belong to the Oswald ripening since the overall area of the LC domain under illumination increases simultaneously. Rice and McConnell [6] once reported a shape transition induced by a photochemical effect. In our case, however, the influence of the photochemical aspects is unlikely. It has been found from the UV vis spectrum of our lipid compound that the absorption band was below $250 \mathrm{~nm}$ [18]. Actually, it has been shown by our previous investigations that the illumination-related domain growth is associated with the mass transport into the illuminated region from an outside area $[16,17]$. The illuminationrelated domain growth arises from an illumination-related local mass transport, which can be attributed to the illumination-induced inhomogeneous distribution of chemical potentials in the illuminated region and the surrounding areas, respectively $[16,17]$. The decomposed fluorescence probes decrease local chemical potential in the illuminated area. The difference of chemical potential across the boundary between the illuminated region and the surrounding unilluminated region is the driving force to move the amphiphilic molecules into the illuminated area. This effect is physically equivalent to a centripetal compressing surface pressure field.

Corresponding to the morphological changes shown in Fig. 1, the dimension of the pattern also increases. The dimension of the fractal-like pattern is determined by the density-density correlation function of the cluster $[19,20]$. Figure 2 illustrates the evolution of the fractal dimension of the LC domain $[D(t)]$ as a function of time. For instance, 


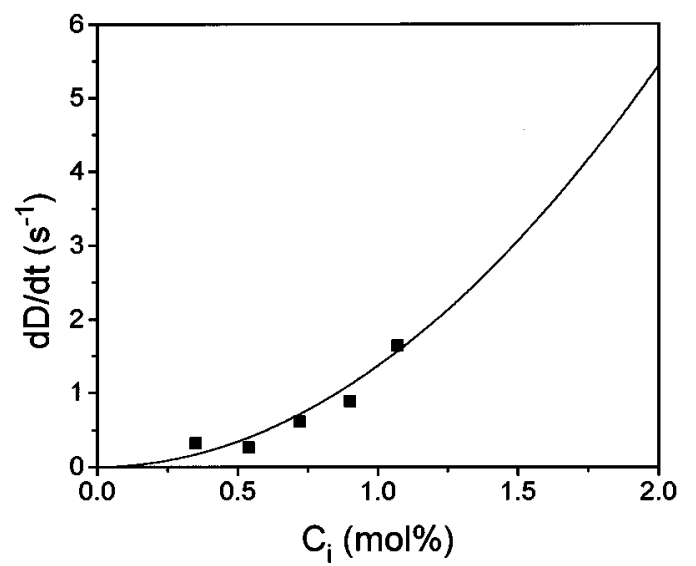

FIG. 3. The plot to show the increasing rate of the fractal dimension as a function of the concentration of the fluorescence probe $C_{i}$. The square dots are the experimental data. The solid line is plotted according to Eq. (6), in which $k_{3}, k_{4}$ are chosen to be 27.0 and $1.1 \times 10^{4}$, respectively.

when the fluorescence probe concentration is $1.07 \% \mathrm{~mol}$, the fractal dimension of the LC domain increases from 1.78 to 1.87 by an illumination of $600 \mathrm{sec}$. Furthermore, our analysis indicates that the increasing rate of the fractal dimension $D(t)$ depends on the concentration of the fluorescence probe (Fig. 3). When the concentration of the fluorescence probe is less than $0.6 \% \mathrm{~mol},(d D(t) / d t)$ increases slowly. When the fluorescence concentration becomes higher, $(d D(t) / d t)$ builds up more rapidly. Figure 4 shows the density-density correlation function of the same growing domain as a function of time. Initially the density-density correlation function of the fractal pattern has a long, straight linear region, which indicates the scaling invariance (or selfsimilarity) of the ramified pattern. After illumination of several hundred seconds, the previous straight region on the plot of the density-density correlation function shrinks and finally the linear region disappears. This implies that the self-

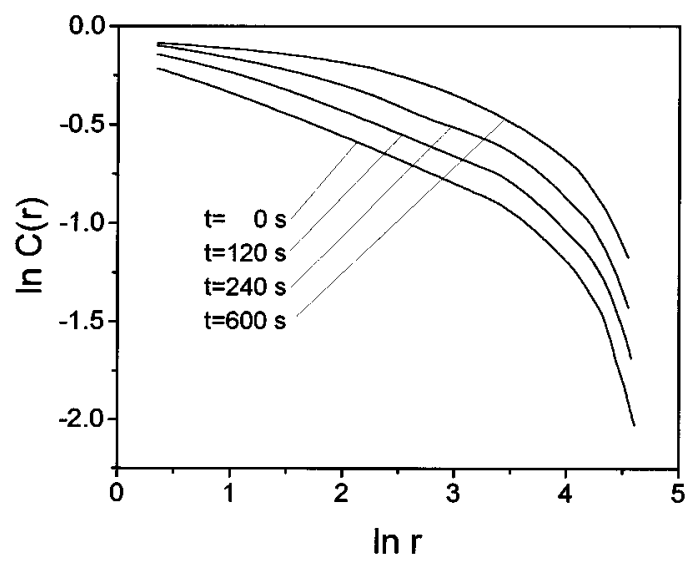

FIG. 4. During the illumination-related domain growth, the property of self-similarity of the fractal domain disappears gradually. This plot illustrates the evolution of the density-density correlation function of a fractal-like pattern. One may find that the initial straight, long, linear region shrinks gradually. After illumination for $600 \mathrm{sec}$. the linear region on the plot of the density-density correlation function is not evident. similarity of the fractal pattern disappears gradually when branches of the domain are thickened.

We are interested in the mechanism of the increase of the fractal dimension during the growth. Concerning the LC domain growth in monolayers, it was generally believed that diffusion-limited aggregation (DLA) [20] played an important role. In the early stage people thought that growth of the fractal-like LC domain depended on the diffusion of the fluorescence probes from the LC domain to the surrounding $\mathrm{LE}$ phase [3]. Whereas it was found later that fractal-like domain can also be formed without fluorescence probes [4]. Therefore, it seems that diffusion of fluorescence probes is not an essential ingredient in the growth of fractal-like LC domains. Figure 3 implies that the domain growth rate increases when the fluorescence concentration is increased. This result is unfavorable to the growth model related to the diffusion of fluorescence probes. Actually, if the model of the diffusion of fluorescence probes were true, one would observe a declining domain growth rate when the fluorescence concentration increases. Suresh, Nittmann, and Rondelez [5] once proposed that diffusion of amphiphilic molecules themselves can be responsible for the growth of the fractal-like domain. However, this model still cannot explain quite well the increasing of the domain dimension during the growth. Based on our experimental observations, we propose a model which includes not only molecular diffusion, but also positional relaxation of molecules, to describe the observed phenomena. Before discussing this model in detail, it will be helpful to have a review of the microscopic picture of phase transitions in the Langmuir monolayer film. When the area of the monolayer film is large enough, the monolayer film is in the gaseous phase, in which the molecular density is so low that the area per molecule is much greater than the molecular dimension. Meanwhile, the hydrocarbon portions of the molecules make a significant contact with the water surface. When the area of the monolayer film is decreased, a transition from the gaseous phase to the liquid-expanded (LE) phase occurs. In the LE phase, hydrocarbon chains lift up from the surface but remain largely disordered. Further compression of the monolayer film leads to the appearance of the LC phase, in which hydrocarbon chains lift up and the degree of the chain alignment is much higher than in the LE phase. Actually, long range orientational order in the LC phase have been found previously [21]. To some extent, LC domains can be considered as $2 \mathrm{D}$ crystals. The LC domain is grown by changing the orientation of amphiphilic molecules, while this is governed by the molecular density in monolayers. In monolayer film, there exists a critical molecular density, beyond which nucleation of the LC phase may occur. The domains of the LC phase grow continuously till the equilibrium of the LE and the LC phases is established. Thereafter, if the molecular density of the LE phase is further increased, the molecules around the ever-grown LC domains will change their orientations to that in the LC phase in order to resume the equilibrium concentration in the LE phase. As we reported earlier $[8,16,17]$, during the illumination-related domain growth amphiphilic molecules are continuously transported into the illuminated region. So the molecular density in the LE phase in the illuminated region may become higher than the equilibrium value. Consequently, LC domains in the illuminated area grow. In the illumination-related domain growth, the 
driving force is much smaller than the case of compression. Therefore, it is quite probable that before being incorporated into the LC phase, molecules may diffuse alone the LE-LC interface until they find positions with the lowest energy. The interfacial diffusion or relaxation is responsible for the thickening of the fractal branches. Therefore, we claim the essential points of the domain growth in this experiments as follows: the local mass transport supplies the nutrient for the illumination-related domain growth, while the surface (interline) relaxation in the growth leads to the morphology transition.

Such a picture of domain growth is supported by our experimental observations. As we discussed in our previous report $[16,17]$, transport of amphiphilic molecules into the illuminated region from the surrounding unilluminated region depends on the molecular interactions between the amphiphilic molecules and the illumination-decomposed fluorescence probes. Stronger molecular interactions may greatly decrease the chemical potential in the illuminated region and hence induce stronger molecular flux into the illuminated region. It is therefore reasonable to expect that when the fluorescence concentration becomes higher, the driving force for molecular transport increases correspondingly. As the result, the growth of the LC domain becomes faster at higher fluorescence concentration, which is implied by Fig. 3.

It is shown in Fig. 3 that $d D / d t$ builds up very slowly when $C_{i}$ is low, while it increases more rapidly when $C_{i}$ becomes higher. This can be understood in the framework of the theory of the regular solution. As we presented previously [16], the difference of the chemical potential in the illuminated region and in the surrounding area $\Delta \mu$ can be expressed as

$$
\Delta \mu=k T \ln \left(\left(1-C_{i}\right) e^{C_{i}^{2} \Sigma_{j} \Phi_{j} / k T}\right)
$$

where $C_{i}$ represents the concentration of fluorescence probe, $\Phi_{j}=\phi_{j}^{A, F}-\frac{1}{2}\left(\phi_{j}^{A, A}+\phi_{j}^{F, F}\right), \quad \phi_{j}^{A, F} \phi_{j}^{A, A}, \phi_{j}^{F, F}$ stand for the interaction energy between the amphiphilic molecules $(A)$ and fluorescence $\operatorname{probe}(F)$, the interaction energy between amphiphilic molecules $(A-A)$ and the interaction energy between fluorescence probes $(F-F)$, respectively. The sum in the definition of $\Delta \mu$ covers $n$ neighboring molecules. The growth of the LC domain in the illuminated region requires negative $\Delta \mu$, which means

$$
\left(1-C_{i}\right) e^{C_{i}^{2} \Sigma_{j} \Phi_{j} / k T}<1
$$

Let $\mathrm{r}(\mathrm{t})$ denote the linear size of a LC domain, then the mass aggregation rate of the LC domain in the illuminated region $d m / d t$ can be expressed as,

$$
\begin{aligned}
\frac{d m}{d t}= & \frac{d\left(\rho r(t)^{D(t)}\right)}{d t}=\rho D(t) r(t)^{D(t)-1} \frac{d r}{d t} \\
& +\rho r(t)^{D(t)} \ln r(t) \frac{d D(t)}{d t}
\end{aligned}
$$

where $(d r(t) / d t)$ is the radial growth rate of the boundary of the LC domain, $\rho$ is the density of fractal-like LC domain. We propose that the radial growth rate be proportional to the difference of chemical potential $\Delta \mu$, i.e.,

$$
\frac{d r(t)}{d t}=k_{1}|\Delta \mu|,
$$

where $k_{1}$ is a constant. Equation (4) implies that the anisotropy in the interfacial (interline) growth is not strong and there is no nucleation barriers in the interfacial growth. On the other hand, the growth rate of the LC domain in the illuminated region $d m / d t$ is proportional to both the length of the boundary of the illuminated region $\left(\sim 2 \pi r_{0}, r_{0}\right.$ is radius of the illuminated region) and $\Delta \mu$, i.e. ,

$$
\frac{d m}{d t}=k_{2} \cdot 2 \pi r_{0}|\Delta \mu|,
$$

where $k_{2}$ is a constant. Taking (4) and (5) into (3), finally we get

$$
\begin{aligned}
\frac{d D(t)}{d t} & =\frac{k_{2} 2 \pi r_{0}-\rho k_{1} D r^{D-1}}{\rho r^{D} \ln r} k T \ln \left(\frac{1}{1-C_{i}} e^{-C_{i}^{2} \Sigma_{j} \Phi_{j} / k T}\right) \\
& =\frac{k_{2} 2 \pi r_{0}-\rho k_{1} D r^{D-1}}{\rho r^{D} \ln r} k T\left(\ln \frac{1}{1-C_{i}}-C_{i}^{2} \sum_{j} \Phi_{j} / k T\right) \\
& =k_{3} \ln \left(\frac{1}{1-C_{i}}\right)+k_{4} C_{i}^{2} .
\end{aligned}
$$

In Eq. (6) $k_{3}$ and $k_{4}$ are constants associated with $\rho$, $k_{1}, k_{2}, k, T, r, D$ and $\Phi_{j}$. Experimentally it has been demonstrated that $(d D / d t)$ is positive. From Eqs. (2) and (6) one may easily find that $k_{2} \cdot 2 \pi r_{0}>\rho k_{1} D r^{D-1}$. Therefore $k_{3}>0$. On the other hand, the definition of $\Phi_{j}$ indicates that $\Phi_{j}$ is negative in most cases, which means that $k_{4}$ is usually positive. One may expand the first item of Eq. (6) and find that at a very low fluorescence concentration, $d D / d t$ is approximately a slowly increasing linear function of $C_{i}$. When $C_{i}$ becomes higher, according to Eq. (6), ( $\left.d D / d t\right)$ increases more rapidly. $d D / d t$ vs $C_{i}$ expressed by Eq. (6) is plotted as the solid line in Fig. 3, which is consistent with the experimental data.
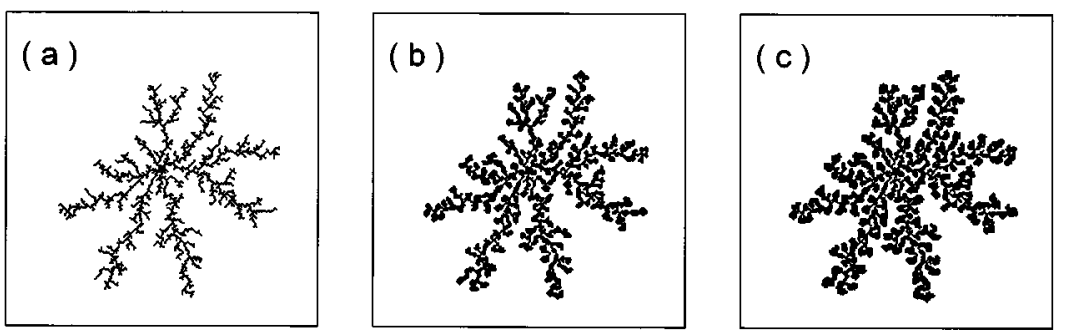

FIG. 5. The Monte Carlo simulated domain growing process based on the model described in the text, in which $l=3$. The domain branches are thickened gradually in this process, and the domain dimension increases correspondingly. The edge of each frame (a)-(c) is 250 lattice long. The number of particles contained in the cluster is (a) 2000; (b) 5000; (c) 7000, respectively. 
It is a challenge to understand why a growing fractal-like domain loses its self-similarity gradually, and more basically, what are the essential ingredients governing the domain morphology. According to the growth model described in the last paragraphs, the domain growth is induced by the flux of amphiphilic molecules continuously diffusing into the illuminated region from the surrounding areas, while interfacial relaxation plays an important role in the thickening of domain branches. We simulate the illumination-related domain growth by a computer. Initially a seed cluster is generated by the standard DLA process [20]. Then an envelope is set $l$ lattice away around the seed cluster. The envelope mimics the shape of the cluster and is continuously adjusted to keep the distance between the fractal-like domain and the envelope at fixed value $l$. Particles are randomly released on the envelope to approach the seed cluster by a random walk. When a particle lands on the cluster, it may relax to the nearest- or the next-nearest- neighbor sites in order to get the lowest potential energy, where the largest local occupation percentage can be found. When $l \rightarrow \infty$ this model is equivalent to the standard model of DLA. In the case of illumination-related domain growth, the molecules do not need to diffuse a long distance before they incorporate into the LC domain. So $l$ should be small. Small $l$ in the domain growth makes the screening effect weaker, but the influence of the screening effect is still observable. The detailed discussion of this growth model will be presented separately. Here we only show part of our simulation results related to the dimension increasing effect in the domain growth. Figure 5 shows the growing process of a LC domain with $l=3$. Clearly the essential features shown in Fig. 1 have been included in Fig. 5, that is, the dimension of the cluster increases gradually when the cluster grows larger.

To summarize, we report in this paper the morphological transition from fractal to nonfractal in the growth of the LC domain in lipid monolayers. The branches of the LC domain are thickened during the growth. We propose that the surface relaxation plays an important role. The surface relaxation may also be responsible for the disappearance of selfsimilarity and the increase of the domain dimension in the domain growth. Our observations also imply that in the case of weak anisotropy, there may be no nucleation barrier or other barriers in the interfacial growth, in other words, the growing interface of the LC domain may be roughened.

\section{ACKNOWLEDGMENTS}

This work was supported by the Natural Science Foundation of China (project No. 19425007) and a grant from the State Science and Technology Commission of China. One of the authors (M.W.) wishes to thank Professor H. Ringsdorf and the Institute of Organic Chemistry of the University of Mainz for conveniently providing the fluorescence microscope. The support from QiuShi Science and Technology Foundation (Hong Kong) is also acknowledged.
[1] C. M. Knobler, Science 249, 870 (1990).

[2] R. M. Weis and H. M. McConnell, Nature 310, 47 (1984).

[3] A. Miller, W. Knoll, and H. Möhwald, Phys. Rev. Lett. 56, 2633 (1986).

[4] D. Hönig and D. Möbius, J. Phys. Chem. 95, 4590 (1991); S. Henon and J. Meunier, Rev. Sci. Instrum. 62, 936 (1991).

[5] K. A. Suresh, J. Nittmann, and F. Rondelez, Europhys. Lett. 6, 437 (1988).

[6] P. A. Rice and H. M. McConnell, Proc. Natl. Acad. Sci. USA 86, 6445 (1989).

[7] M. Yoneyama, et al., J. Phys. Chem. 97, 5124 (1993).

[8] Mu Wang et al., Phys. Rev. Lett. 71, 4003 (1993).

[9] A. Buka, P. Palffy-Muhoray, and Z. Rácz, Phys. Rev. A 36, 3984 (1987).

[10] E. Ben-Jacob and P. Garik, Nature 343, 523 (1990).

[11] Mu Wang and Nai-ben Ming, Phys. Rev. Lett. 71, 113 (1993).

[12] A. Hill, Nature 348, 426 (1990).

[13] P. E. Cladis, J. T. Gleeson, and P. L. Finn, in Nonlinear Struc- tures in Physical Systems: Pattern Formation, Chaos, and Waves (Springer-Verlag, New York, 1990), p. 56; P. E. Cladis, J. T. Gleeson, P. L. Finn, and H. R. Brand, Phys. Rev. Lett. 67, 3239 (1991).

[14] A. Miller and H. Möhwald, Europhys. Lett. 2, 67 (1986).

[15] H. M. McConnell, J. Phys. Chem. 94, 4728 (1990); P. Muller and F. Gallet, ibid. 95, 3257 (1991); K. J. Stine, C. M. Knobler, and R. C. Desai, Phys. Rev. Lett. 65, 1004 (1990).

[16] Mu Wang et al., Phys. Lett. A 203, 235 (1995).

[17] Mu Wang et al., Phys. Rev. E 53, 2580 (1996).

[18] R. J. M. Nolte (private communication); see also, Jan van Esch, Ph.D. thesis, Nijmegen University, The Netherlands, 1993 (unpublished).

[19] T. Viseck, Fractal Growth Phenomena (World Scientific, Singapore, 1989).

[20] T. A. Witten and L. M. Sander, Phys. Rev. Lett. 47, 1400 (1981).

[21] For instance, K. Kjaer et al., Phys. Rev. Lett. 58, 2224 (1987). 